\title{
Two TP53 germline mutations in a classical Li-Fraumeni syndrome family
}

\author{
Liselotte P. van Hest • Mariëlle W. G. Ruijs • \\ Anja Wagner - Conny A. van der Meer · \\ Senno Verhoef - Laura J. van't Veer · \\ Hanne Meijers-Heijboer
}

Received: 8 December 2006/ Accepted: 18 December 2006/Published online: 23 February 2007

(C) Springer Science+Business Media B.V. 2007

\begin{abstract}
Li-Fraumeni syndrome (LFS) is an autosomal dominantly inherited cancer predisposition syndrome characterized by a combination of tumors including sarcoma, breast cancer, brain tumors, adrenocortical carcinoma and leukemia. Germline mutations in the tumor suppressor gene TP53 are associated with LFS. We present a family with LFS in which initially a novel germline TP53 intron 5 splice site mutation was found. A second germline TP53 mutation, the exon 7 Asn235Ser $(704 \mathrm{~A} \rightarrow \mathrm{G})$ mutation, was detected in this family through pre-symptomatic DNA testing. This latter mutation has been reported repeatedly in the literature as a pathogenic mutation involved in LFS. We provide evidence for pathogenicity of the novel intron 5 splice site mutation, whereas this evidence is lacking for the exon 7 Asn235Ser $\quad(704 \mathrm{~A} \rightarrow \mathrm{G})$ mutation. Our findings emphasize the importance of performing additional tests in case of germline sequence variants with uncertain functional effects.
\end{abstract}

Liselotte P. van Hest and Mariëlle W.G. Ruijs contributed equally to this work.

L. P. van Hest - A. Wagner - C. A. van der Meer .

H. Meijers-Heijboer

Department of Clinical Genetics, Erasmus Medical Center, Rotterdam, The Netherlands

M. W. G. Ruijs · S. Verhoef · L. J. van't Veer Family Cancer Clinic, The Netherlands Cancer Institute, Amsterdam, The Netherlands

M. W. G. Ruijs · H. Meijers-Heijboer $(\square)$

Department of Clinical Genetics and Human Genetics, VU

University Medical Center, Amsterdam, The Netherlands

e-mail: h.meijers@vumc.nl
Keywords Functional assay $\cdot \mathrm{Li}$-Fraumeni syndrome . TP53 germline mutations - Unclassified variants

\section{Introduction}

Li-Fraumeni syndrome (LFS) was first described in 1969 [1] as a hereditary cancer predisposition syndrome characterized by the occurrence of bone and soft tissue sarcoma, breast cancer, brain tumors, adrenocortical carcinoma and leukemia. Germline mutations in the TP53 tumor suppressor gene on chromosome $17 \mathrm{p} 13$ were associated with LFS in 1990 [2]. About $75 \%$ of clinical LFS-families carry a TP53 germline mutation, and $40 \%$ of families with the less stringent criteria of Li-Fraumeni-like syndrome (LFL) [3]. So far, 283 different pathogenic germline mutations have been reported of which $74 \%$ are missense mutations and $4 \%$ splice site mutations [4]. LFS is a very rare disease, and therefore it is not surprising that thus far only one family with more than one germline mutation has been reported [5]. Quesnel et al. described a child with a rhabdomyosarcoma at two years of age and a brain tumor at age 10 who carried three different TP53 germline alterations $(\mathrm{R} 290 \mathrm{H}$ on one allele and R156H/R267Q on the other allele). Individual analysis of each mutant indicated that they separately have either a weak mutant phenotype or no mutant phenotype at all. However, the R156H/R267Q double mutant had a strong mutant behavior $[5,6]$.

TP53 knock out mice are viable and are usually born without any observable gross defects, but then rapidly develop a variety of tumors, including sarcomas and other tumors commonly seen in LFS [7-9]. 
We here describe a classical LFS family with two germline TP53 mutations; one novel splice site mutation, and one missense mutation that had been classified as a pathogenic germline mutation before.

\section{Patients and methods}

The family (Fig. 1) was of Dutch ancestry and presented at the department of Clinical Genetics at Erasmus MC, Rotterdam. Family history data were confirmed through medical records and pathology reports. Informed consent was obtained from the patients or from a first degree relative in case the patient was deceased. DNA was isolated from peripheral lymphocytes according to standard procedures. From deceased affected family members DNA was extracted from paraffin embedded blocks of the tumor. Screening for TP53 germline mutations was performed by sequence analysis of all coding exons (2-11) and flanking intron-exon boundaries. The functional effect of the germline mutations was examined by FASAY, a yeast-based assay studying the biological transcriptional ability of p53 [10]. In a control group of 150 anonymous blood donors the presence of both mutations was analyzed by denaturing gel electrophoresis (DGGE). Immunohistochemical staining was performed in seven affected family members to assess the presence of the $\mathrm{p} 53$ protein, using the mouse monoclonal antibody DO7, according to standard procedures. (Dako, Glostrup, Denmark). Two splice site prediction programs were applied to examine the two mutations (NetGene2 Server and BDGP splice site prediction by Neural Network [11, 12]). The conservation throughout evolution of the mutational spots and the polarity status of the normal and mutated amino acids were studied. Two TP53 mutation-databases were checked: the IARQ TP53 database [4] and the p53 Soussi mutation database cancer $[4,13]$.

\section{Results}

The pedigree is depicted in Fig. 1 and further clinical details are outlined in Table 1 . The family was clinically diagnosed with Li-Fraumeni syndrome. Based on the family history, it seemed likely that individual II-10 might have had a de novo TP53 mutation, which he subsequently had passed to the majority of his offspring (individual II-10 and his offspring, further called core-family). Another hypothesis would be the presence of a germline mosaicism in individual II-10 or his spouse.

A novel intron 5 splice site mutation (IVS5-1 $\mathrm{G} \rightarrow \mathrm{A}$ ) was found in the index patient (pedigree number III-4). This TP53 mutation segregated with the disease; the 5 affected family members of the core family of whom DNA was available were carriers (pedigree numbers II-10, III-4, III-7, III-8 and III-12). The mutation was inherited paternally. The sister of the father who developed pancreatic cancer at 69 years of age (pedigree number II-1) did not carry the intron 5

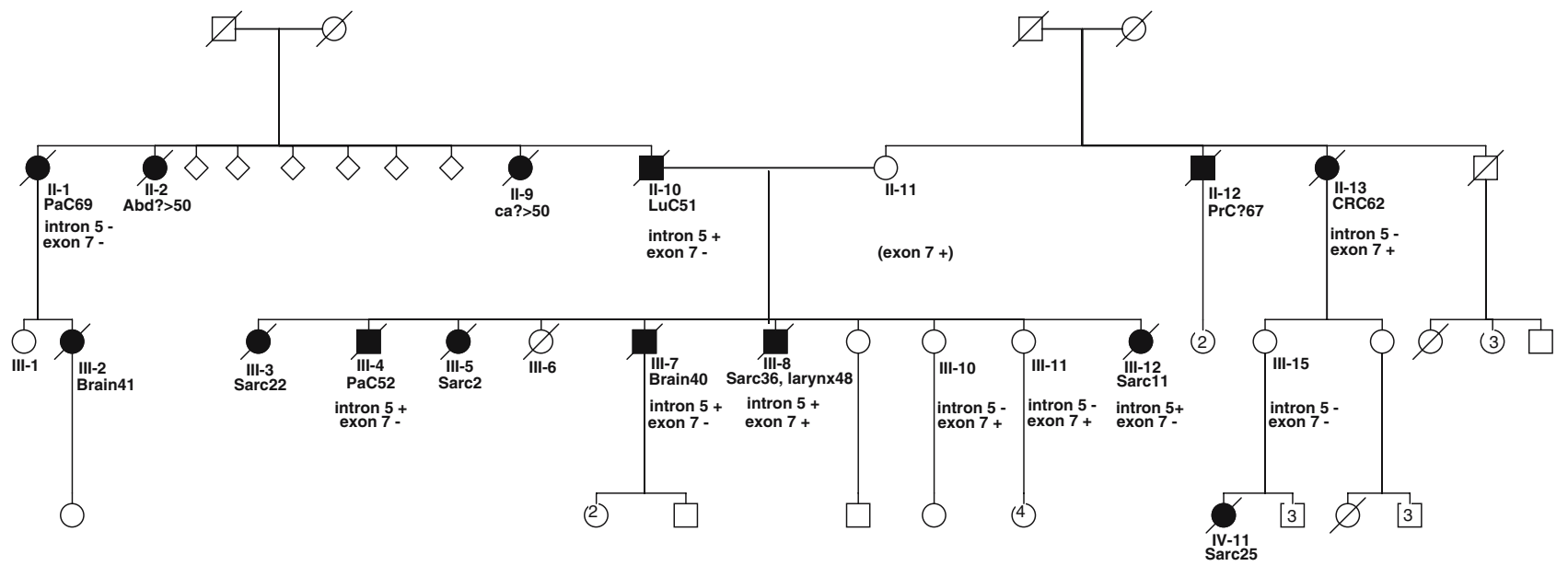

Fig. 1 Pedigree of the studied family: Square symbols indicate males, round symbols indicate females, diamond symbols indicate individuals of unknown sex, line across symbol means deceased individual. Tumor type and age at diagnosis of the tumors are indicated below the individual identifiers. When a question mark follows the diagnosis, this indicates affected individuals with diagnosis by family history, all other diagnosis are confirmed by pathology reports. $\mathrm{PaC}=$ pancreatic cancer, Abd = abdominal cancer, ca = cancer of unknown origin, $\mathrm{LuC}=$ lung cancer, $\operatorname{PrC}=$ prostate cancer, $\mathrm{CRC}=$ colorectal cancer, $\quad$ Brain $=$ brain tumor, $\quad$ Sarc $=$ sarcoma, Larynx $=$ laryngeal carcinoma, intron 5 and exon 7 are the two different mutations tested for, $+=$ mutation present, $-=$ mutation absent 
Table 1 Clinical data and mutation analysis

\begin{tabular}{|c|c|c|c|c|}
\hline Patient & Diagnosis & $\begin{array}{l}\text { Age of } \\
\text { onset }\end{array}$ & Intron 5: IVS 5-1G > A & Exon 7: Asn235Ser $(704 \mathrm{~A} \rightarrow \mathrm{G})$ \\
\hline II-1 & pancreatic adenocarcinoma & 69 & $-(\mathrm{t})$ & $-(\mathrm{t})$ \\
\hline II-2 & abdominal cancer, not verified & $>50$ & & \\
\hline II-9 & cancer, not verified & $>50$ & & \\
\hline II-10 & small cell lung carcinoma & 51 & $+(\mathrm{t})$ & $-(\mathrm{t})$ \\
\hline II-11 & healthy, 83 years of age & & not tested & not tested, obligate carrier \\
\hline II-12 & prostate cancer, not verified & 67 & & \\
\hline II-13 & adenocarcinoma of colon transversum & 62 & $-(\mathrm{t})$ & $+(\mathrm{t})$ \\
\hline III-2 & oligodendroglioma & 41 & & \\
\hline III-3 & osteosarcoma of the humerus & 22 & $\begin{array}{l}\text { material not suitable for } \\
\text { analysis }\end{array}$ & $\begin{array}{l}\text { material not suitable for analysis, } \\
\text { suspicion wild type }\end{array}$ \\
\hline III-4 & pancreatic adenocarcinoma & 52 & $+(b)$ & $-(\mathrm{b})$ \\
\hline III-5 & lymphosarcoma of the kidney & 2 & & \\
\hline III-6 & $\begin{array}{l}\text { this girl died at the age of twelve months, } \\
\text { reason unknown }\end{array}$ & & & \\
\hline III-7 & anaplastic oligodendroglioma & 40 & $+(\mathrm{t})$ & $-(\mathrm{t})$ \\
\hline III-8 & $\begin{array}{l}\text { leiomyosarcoma right leg laryngeal } \\
\text { carcinoma }\end{array}$ & 36 & $+(\mathrm{t})$ & $+(\mathrm{t})$ \\
\hline III-10 & healthy, 51 years of age & 48 & $-(b)$ & $+(\mathrm{b})$ \\
\hline III-11 & healthy, 47 years of age & & $-(\mathrm{b})$ & $+(\mathrm{b})$ \\
\hline III-12 & rabdomyosarcoma, retroperitoneal & 11 & $+(\mathrm{t})$ & $-(\mathrm{t})$ \\
\hline III-15 & healthy, 67 years of age & & $-(\mathrm{b})$ & $-(\mathrm{b})$ \\
\hline IV-x & healthy, 29 years of age & & $+(b)$ & $-(b)$ \\
\hline IV-11 & leiomyosarcoma of the face & 25 & not tested & not tested \\
\hline
\end{tabular}

Not verified $=$ diagnosis by family history, $>50=$ age of onset over 50 years, $\mathrm{t}=$ tested on DNA isolated from tissue, $\mathrm{b}=$ tested on DNA isolated from blood, $+=$ mutation present, $-=$ mutation absent, not tested $=$ mutation analysis not performed

mutation. One healthy individual was shown to be a carrier at the age of 29 years.

FASAY analysis showed that the intron 5 mutation lacks biological transcriptional activity (photo of data not shown). In 300 control alleles the intron 5 germline mutation was not found. Immunohistochemical staining for p53 was negative in all tumors of the carriers of the intron 5 mutation. A phenomenon more frequently observed for TP53 splice site mutations. Two splice site prediction programs confirmed that the effect of this mutation is splicing out of exon 6 , leading to a frameshift with a transcriptional stop early in exon 7 . The IVS5-1 affects a $100 \%$ conserved splice acceptor site.

The exon 7 missense mutation, Asn235Ser $(704 \mathrm{~A} \rightarrow \mathrm{G})$, was initially detected in a presymptomatic test of relative III-10. The mutation did not segregate with the disease as 4 out of 5 cancer patients from the core-family did not carry this mutation, while three healthy women were found carriers at the ages of 47, 51 and 83 years (pedigree numbers III-10, III-11 and II-11, the last one being an obligate carrier). The single affected carrier in the core family (pedigree number III-8) developed a sarcoma at 36 years and laryngeal carcinoma at 48 years. The Asn235Ser mutation turned out to be maternally transmitted as individual II-13 carried this mutation. She developed colorectal cancer at 62 years of age. Interestingly, the healthy daughter of this woman (pedigree number III15), who's daughter died of a leiomyosarcoma of the face, tested negative for the exon 7 mutation. FASAY analysis showed that the Asn235Ser mutation had normal transcriptional activity (photo of data not shown). In 300 control alleles this mutation was found once. Immunohistochemical staining for p53 was negative in tumor material of both affected mutation carriers, including the tumor of the patient with both germline mutations (pedigree number III-8). Two different splice site prediction programs were unanimous in their prediction that the Asn235Ser mutation did not create a cryptic splice site. Orthologous, the Asparagine on this spot is conserved in mouse and rat but not in certain fish. Of note, some fish have a Serine instead of Asparagine at this spot. Paralogous, the Asparagine is conserved in TP51, TP63 and TP73. The polarity of the amino acids Asparagine and Serine is similar. The Asn235Ser mutation was reported 5 times in the TP53 germline mutation databases screened.

\section{Discussion}

We here present a LFS family with, at first sight, two pathogenic germline TP53 mutations. Additional tests, 
however, showed that one of them was highly unlikely to be causative to the disease phenotype.

The novel TP53 intron 5 splice site mutation (IVS5-1 G > A) was first detected. We considered this mutation causative to LFS in view of its co-segregation with the 5 affected cases in the core-family, its functional consequence (stop of transcription early in exon 7 ), and the $100 \%$ conservation of this splice acceptor site throughout evolution. We therefore offered the family presymptomatic testing for this mutation. In the process, surprisingly, a missense mutation in exon 7 Asn235Ser (704A $\rightarrow \mathrm{G})$ was detected, which had been classified before as a pathogenic germline mutation in multiple reports (see Table 2) [14-18]. In order to provide meaningful diagnostic genetic testing within this family, we defined the predicted contribution of each of the mutations to the disease phenotype in more detail. In summary, all data obtained on the novel intron 5 mutation pointed towards a causative association of this mutation with LFS within the core-family.

In contrast, the exon 7 Asn235Ser mutation did not segregate with disease in the core-family as only 1 out of 5 affected cases carried this mutation while three healthy individuals were found carriers at ages 47,51 and 83 years. Of note, a third-degree relative of the core-family who died of leiomyosarcoma at age 25 years was also excluded as a carrier. The immunohistochemical staining of the two tumors of carriers of the Asn235Ser showed no expression of p53, while positive staining is commonly seen for a pathogenic missense mutation [19]. Although generally codon 100-300 is called the DNA binding domain, codon 235 is not directly involved in DNA-binding; it is located in between two domains that interact extensively to provide DNA contacts [20]. Therefore, this mutation was likely not to affect the DNA-binding properties of $\mathrm{p} 53$. Indeed, normal results, DNA-binding properties and transcription activation, were obtained by FASAY.

The Asn235Ser mutation has been reported in the germline five times before (Table 2) [14-18]. None of the authors of these reports found the mutation in a classical LFS family. The authors unfortunately performed no functional assays, or determined its prevalence in healthy controls.

Still, three out of these five reports classified the mutation as pathogenic. Diller et al. [14] and Auer et al. [17] based their conclusion on the fact that the mutation had been described as a somatic mutation in cancer before. Huusko et al. [18] described a LFL family with this mutation. The predominant cancer type in this family was breast cancer and no BRCA1 or $B R C A 2$ mutation was identified. They claimed the mutation to be pathogenic on the basis of results in the tumors regarding loss of heterozygosity of the TP53 locus and p53 immunohistochemistry, and on the fact that the mutation had been associated with cancer predisposition before by Diller et al.[14] and Cornelis et al.[15]. To note, the mutation did cosegregate; two out of three patients in this family were tested. Both were carriers, however, also two healthy adults were carrier. Ponten et al. [16] concluded the mutation to be a rare polymorphism. He found the Asn235Ser in a 72 year old male with two basal cell carcinoma's in which he found also two somatic TP53 mutations. Cornelis et al. [15] recommended functional assays to determine the pathogenic nature of this mutation.

Soussi et al. [6] studied all somatic TP53 mutations of the TP53 mutation database (http://p53.curie.fr) by using very extended functional assays. They analyzed the transactivation activity of the mutations with respect to eight promotors and compared the activity to p53 wild type (wt) activity. The Asn235Ser mutation was described 14 times as a somatic mutation, 6 times in combination with another somatic mutation. The mean activity of Asn235Ser on 8 promotors was $86 \%$ of wt activity, well above their cut off point for pathogenic mutations ( $<20 \%$ of wt activity).

Besides the fact that Asparagine is conserved in paralogs, all data provide evidence that Asn235Ser is a rare polymorphism or at best a low penetrance allele rather than a pathogenic mutation for LFS. It is remarkable that this mutation is found often in combination with another (either somatic or germline) mutation.

Our case report illustrates potential pitfalls in clinical genetic testing for cancer susceptibility. In order to provide optimal accurate risk assessment in cancer susceptibility testing, critical literature study is a prerequisite. We showed the importance of confirmation of carrier status of all affected family members once a pathogenic mutation within the family is found. Also, in case of sequence variants with uncertain functional consequences, additional tests are mandatory before genetic testing is offered in clinical settings. 


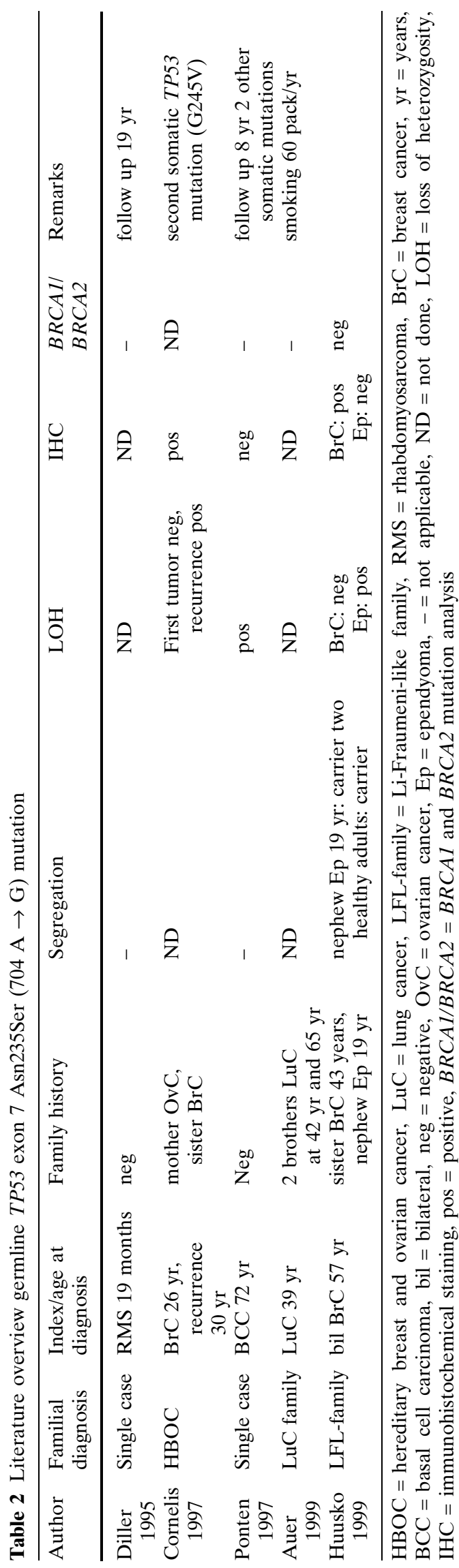

\section{Electronic-database information}

NetGene2 server (splice site finder), www.cbs.dtu.dk./ services/NetGene2/

BDGP splice site prediction by Neural Network, www.fruitfly.org/seq_tools/splice.html

IARQ TP53 database, http://www-p53.iarc.fr/

p53 Soussi mutation database cancer, http:// p53.free.fr/

\section{References}

1. Li FP, Fraumeni JF Jr (1969) Soft-tissue sarcomas, breast cancer, and other neoplasms. A familial syndrome? Ann Intern Med 71:747-752

2. Malkin D, Li FP, Strong LC et al (1990) Germ line p53 mutations in a familial syndrome of breast cancer, sarcomas, and other neoplasms. Science 250:1233-1238

3. Varley JM (2003) Germline TP53 mutations and Li-Fraumeni syndrome. Hum Mutat 21:313-320

4. Olivier M, Eeles R, Hollstein M et al (2002) The IARC TP53 database: new online mutation analysis and recommendations to users. Hum Mutat 19:607-614

5. Quesnel S, Verselis S, Portwine C et al (1999) p53 compound heterozygosity in a severely affected child with Li- Fraumeni syndrome. Oncogene 18:3970-3978

6. Soussi T, Kato S, Levy PP et al (2005) Reassessment of the TP53 mutation database in human disease by data mining with a library of TP53 missense mutations. Hum Mutat 25:6-17

7. Donehower LA, Harvey M, Slagle BL et al (1992) Mice deficient for p53 are developmentally normal but susceptible to spontaneous tumours. Nature 356:215-211

8. Purdie CA, Harrison DJ, Peter A et al (1994) Tumour incidence, spectrum and ploidy in mice with a large deletion in the p53 gene. Oncogene 9:603-609

9. Sah VP, Attardi LD, Mulligan GJ et al (1995) A subset of p53-deficient embryos exhibit exencephaly. Nat Genet 10:175-180

10. Flaman JM, Frebourg T, Moreau V et al (1995) A simple p53 functional assay for screening cell lines, blood, and tumors. Proc Natl Acad Sci USA 92:3963-3967

11. Hebsgaard SM, Korning PG, Tolstrup N et al (1996) Splice site prediction in Arabidopsis thaliana pre-mRNA by combining local and global sequence information. Nucleic Acids Res 24:3439-3452

12. Celniker SE, Wheeler DA, Kronmiller B et al (2002) Finishing a whole-genome shotgun: release 3 of the Drosophila melanogaster euchromatic genome sequence. Genome Biol 3: RESEARCH0079

13. Beroud C, Soussi T (2003) The UMD-p53 database: new mutations and analysis tools. Hum Mutat 21:176-181

14. Diller L, Sexsmith E, Gottlieb A et al (1995) Germline p53 mutations are frequently detected in young children with rhabdomyosarcoma. J Clin Invest 95:1606-1611

15. Cornelis RS, van Vliet M, van de Vijver MJ et al (1997) Three germline mutations in the TP53 gene. Hum Mutat 9:157-163

16. Ponten F, Berg C, Ahmadian A et al (1997) Molecular pathology in basal cell cancer with p53 as a genetic marker. Oncogene 15:1059-1067 
17. Auer H, Warncke K, Nowak D et al (1999) Variations of p53 in cultured fibroblasts of patients with lung cancer who have a presumed genetic predisposition. Am J Clin Oncol 22:278-282

18. Huusko P, Castren K, Launonen V et al (1999) Germ-line TP53 mutations in Finnish cancer families exhibiting features of the Li-Fraumeni syndrome and negative for BRCA1 and BRCA2. Cancer Genet Cytogenet 112:9-14
19. Lane DP (1994) On the expression of the p53 protein in human cancer. Mol Biol Rep 19:23-29

20. Cho Y, Gorina S, Jeffrey PD et al (1994) Crystal structure of a p53 tumor suppressor-DNA complex: understanding tumorigenic mutations. Science 265:346-355 